\title{
Potencial atrator de tubarões costeiros em recife artificial no litoral norte do estado do Rio de Janeiro, Brasil
}

\author{
Vicente Vieira Faria ${ }^{1,2}$ \\ Ronaldo Novelli ${ }^{1}$ \\ Marcelo Paes Gomes ${ }^{1}$ \\ Ilana Rosental Zalmon ${ }^{1,3}$
}

\begin{abstract}
Attractive potential of coastal sharks in artificial reef on the Northern Coast of Rio de Janeiro State, Brazil. Artificial reefs have been used in many countries to increase coastal fishery productivity. In order to increase fish attraction to the Northern Coast of Rio de Janeiro State, it was installed an artificial reef $(1,500$ $\mathrm{m}^{2}$ ) 5 nautical miles off Manguinhos's Bay (São Francisco de Itabapoana, Rio de Janeiro). The artificial structures were made of tires, concrete and cement blocks. A gill net of $125 \times 3 \mathrm{~m}$ was monthly used in the artificial reef (AR) and in a control area (CA) to determine the effect of the experimental structures on the stock and diversity of coastal sharks. Considering the complexity of a sustainable elasmobranch exploitation, sharks were focused in this study. During 24 months of investigation (April/96 to March/98), a total of 325 individuals distributed in four shark species were captured in the two areas (AR and CA): Mustelus higmani (Springer \& Lowe, 1963) $(\mathrm{AR}=70$; $\mathrm{CA}=82$ individuals), Rhizoprionodon lalandii (Valenciennes, 1839) $(\mathrm{AR}=86 ; \mathrm{CA}$ $=56$ individuals $), R$. porosus (Poey, 1861) $(\mathrm{AR}=16 ; \mathrm{CA}=14$ individuals $)$ and Carcharhinus brachyurus (Günther, 1870) ( $\mathrm{AR}=1$ individual). The attractive potential of the artificial reef is suggested by the predominance of the shark $R$. lalandii in the reef complex after the first year of monitoring, with the increase of the structures. KEY WORDS. Artificial reefs, coastal sharks, Rio de Janeiro State Northern Coast
\end{abstract}

Recifes artificiais têm sido utilizados em várias partes do mundo como uma ferramenta para promover o incremento da ictiofauna (D'ITRI 1986; AMBROSE \& SWARBRICK 1989). Tubarões atraidos por estas estruturas são principalmente espécies naturalmente associadas a ambientes rochosos (BOMBACE et al. 1994; MCGLENNON \& BRANDEN 1994).

A relação entre tubarões e recifes artificiais está baseada na interação entre sua biologia e as características do recife. Fatores como o tipo, tamanho e tempo de imersão do recife artificial e o grau de associação a ambientes recifais naturais de uma dada espécie de tubarão costeiro, podem ocasionar um aumento ou diminuição em sua abundância numa região (AMBROSE \& SWARBRICK 1989; HAROUN et al. 1994; SANTOS \& MonTEIRO 1997, 1998; BAQueIro-CARdenas et al. 1999). Isto se deve a capacidade de mudar o uso de um habitat costeiro em função de disponibilidade de alimento, fuga de predadores, etc. (MORRISEY \& GRUBER 1993).

1) Laboratório de Ciências Ambientais, Centro de Biociências e Biotecnologia, Universidade Estadual do Norte Fluminense. Avenida Alberto Lamego 2000, 28015-620 Campos dos Goytacazes, Rio de Janeiro, Brasil.

2) E-mail: vicentefaria@bol.com.br

3) E-mail: ilana@uenf.br 
Os tubarões têm como características uma maturação tardia, baixo potencial reprodutivo e ciclo de vida longo. Isto os torna particularmente suscetíveis à sobrepesca, em relação a muitas espécies de peixes teleósteos (HOLDEN 1974; STEVENS et al. 2000). Dados da Food and Agricultural Organisation (FAO) indicam uma grande elevação em sua captura e tal esforço de pesca tem provocado um declínio populacional em várias partes do mundo, incluindo a costa brasileira (BONFIL 1994).

Considerando-se que a implantação de recifes artificiais pode elevar o esforço de pesca sobre as populações locais de tubarões costeiros, este estudo teve como objetivo observar as respostas destas espécies à instalação de um recife artificial no litoral norte do estado do Rio de Janeiro.

\section{MATERIAL E MÉTODOS}

\section{Área de estudo}

A região entre o estado do Espírito Santo e a cidade de Cabo Frio - Rio de Janeiro é considerada de grande importância na distribuição zoogeográfica no Atlântico Sul, sendo indicada como o início de transição entre as faunas nectônicas tropical e subtropical (PALACIO 1982; FLOETER \& SOARES-GOMES 1999). Inserida nesta área, a região norte do estado do Rio de Janeiro justifica a condição de zona de transição, sendo banhada ao norte por águas oligotróficas da Corrente do Brasil e influenciada ao sul pela ressurgência da Água Central do Atlântico Sul (VALENTIN \& MONTEIRO-RIBAS 1993; EKAU \& KNOPPERS 1999).

A área de estudo é tipicamente caracterizada pela escassez de costões rochosos. Vasta área do fundo é constituída por lama fina ou areia compacta, ao passo que fundos rochosos são pontuais. A plataforma continental interna é afetada pelo aporte de água e sedimentos dos rios Itabapoana e Paraíba do Sul, fazendo com que a região possua características estuarinas.

\section{Montagem do recife artificial}

Em março de 1996, o recife artificial foi instalado a cinco milhas náuticas da costa de Manguinhos, São Francisco de Itabapoana, Rio de Janeiro (21 $27^{\text {' S; }}$ $\left.41^{\circ} 00^{\prime} \mathrm{W}\right)$, ocupando uma área de $1500 \mathrm{~m}^{2}$. Imerso a cerca de $9 \mathrm{~m}$ de profundidade, o complexo recifal é constituído por três séries de diferentes substratos: 12 colunas de pneus (borracha), 12 manilhas (concreto) e quatro colunas de tijolos (cimento), totalizando 28 módulos experimentais, dispostos de forma variada. Em fevereiro de 1997, foram adicionadas 15 estruturas de reposição, sendo estas oito colunas de pneus e sete tanques pré-fabricados (cimento), diversificando e ampliando o recife.

\section{Amostragem e procedimento de laboratório}

Ao longo de dois anos de estudo, abril/1996 a março/1998, a avaliação do efeito do recife no estoque e na diversidade de tubarões costeiros foi realizada utilizando-se mensalmente redes de espera de fundo de $125 \times 3 \mathrm{~m}$ com malhas de 20, 30, 40, 50 e $60 \mathrm{~mm}$ entre nós adjacentes no recife artificial (RA) e em área controle $(\mathrm{AC})$ próxima, distante cerca 0,6 milhas náuticas. As redes permaneciam submersas em ambos os sítios por cerca de 24 horas. A utilização da AC permitiu a comparação entre o ambiente marinho natural e sob a influência do RA. 
A identificação dos elasmobrânquios seguiu COMPAGNO (1984). Registrouse o comprimento total $(\mathrm{mm})$ e o peso total $(\mathrm{kg})$ de cada espécime.

\section{Análise dos dados}

O RA e a AC foram caracterizados através do número de indivíduos e peso das espécies capturadas, sendo a significância das diferenças entre os dados mensais do primeiro e segundo ano de estudo (antes e depois do incremento das estruturas em fevereiro/1997), testadas pelo teste U-Mann-Whitney (ZAR 1999). Os meses que não apresentaram capturas em ambos sítios foram excluídos de cada análise.

Com relação à diversidade de espécies baseada no número de indivíduos e peso, foi utilizado o índice de Shannon (H'). Em termos de estrutura taxonômica e abundância relativa, os dois grupamentos (RA e AC) foram comparados, através do coeficiente de similaridade quantitativo de Renkonen para determinar o grau de afinidade entre as áreas.

\section{RESULTADOS}

Ao longo de dois anos de estudo, foram registradas quatro espécies de tubarões costeiros no recife artificial (RA) e na área controle (AC) (Tab. I).

Mustelus higmani (Springer \& Lowe, 1963) (Triakidae) foi a espécie mais capturada, não havendo diferença significativa entre as duas áreas, com 82 e 70 indivíduos na $\mathrm{AC}$ e no RA, respectivamente (Tab. I). Já Rhizoprionodon lalandii (Valenciennes, 1839) (Carcharhinidae) foi significativamente $(\mathrm{p}=0,012)$ mais capturado no RA após o incremento de estruturas no recife artificial em fevereiro de 1997 (Tab. II). Rhizoprionodon porosus (Poey, 1861) teve baixa representatividade, com 16 e 14 indivíduos no RA e na $\mathrm{AC}$, respectivamente. Apenas um indivíduo de Carcharhinus brachyurus (Günther, 1870) foi capturado neste estudo (Tab. I).

Tabela I. Tubarões costeiros capturados no recife artificial e na área controle (abr/96 a mar/98).

\begin{tabular}{|c|c|c|c|c|c|}
\hline \multirow{2}{*}{ Familia } & \multirow{2}{*}{ Espécie } & \multicolumn{2}{|c|}{ Recife artificial } & \multicolumn{2}{|c|}{ Área controle } \\
\hline & & Número de individuos & Peso $(\mathrm{kg})$ & Número de individuos & Peso $(\mathrm{kg})$ \\
\hline Triakidae & Mustelus higmani & 70 & 15,98 & 82 & 14,74 \\
\hline \multirow[t]{3}{*}{ Carcharhinidae } & Rhizoprionodon lalandii & 86 & 35,70 & 56 & 28,58 \\
\hline & Rhizoprionodon porosus & 16 & 7.93 & 14 & 5,97 \\
\hline & Carcharhinus brachyurus & 1 & 3,94 & 0 & 0 \\
\hline
\end{tabular}

Analisando-se os valores de peso das duas espécies mais capturadas, não se observou diferença significativa entre as áreas, embora valores totais superiores tenham sido registrados no RA: $R$. lalandii com 35,7 (RA) e 28,6 kg (AC) e $M$. higmani com 16,0 (RA) e 14,7 kg (AC). Apesar disso, evidenciou-se uma maior captura em peso de $R$. lalandii no RA a partir do incremento de estruturas em fevereiro/1997, comparando-se com a área controle (Tab. II).

Com relação à diversidade ( $\left.\mathrm{H}^{\prime}\right)$ baseada no número de indivíduos, foram observados valores reduzidos para ambas as áreas, em função do baixo número de espécies, variando no RA de 0,62 (verão/97) a 1,06 (primavera/97) e, na AC de 0 
(verão/98) a 1,08 (outono/97) (Fig. 1A). Pode-se observar uma variação mais estreita no RA, com uma linearidade dos valores no primeiro ano de estudo, em torno de 0,67 e uma tendência crescente a partir do segundo ano, decorrente de uma distribuição mais homogênea das três espécies capturadas. Já na AC foi observada uma maior variação, sem um padrão de distribuição, com valores superiores nas primaveras 96/97 e outono/97 (Fig. 1B).

Tabela II. Resultados do Teste U-Mann-Whitney comparando a variação do número de individuos e peso total mensal de Rhizoprionodon lalandii ao longo do primeiro e segundo anos de imersão do recife artificial (RA) e em área controle ( $A C)$.

\begin{tabular}{|c|c|c|c|c|c|c|c|}
\hline \multirow{2}{*}{ Ano } & \multirow{2}{*}{ Mês/Ano } & \multicolumn{2}{|c|}{ Número de individuos } & \multirow{2}{*}{$\begin{array}{l}\text { Valor de } p \\
\text { RA } \times A C\end{array}$} & \multicolumn{2}{|c|}{ Peso $(\mathrm{kg})$} & \multirow{2}{*}{$\frac{\text { Valor de } F}{\text { RA } \times A C}$} \\
\hline & & RA & $A C$ & & RA & $A C$ & \\
\hline \multirow[t]{8}{*}{ Primeiro } & Abril/1996 & 7 & 2 & & 2,78 & 0,96 & \\
\hline & Maio/1996 & 4 & 5 & & 2,51 & 2,89 & \\
\hline & Junho/1996 & 2 & 1 & & 0,57 & 0,24 & \\
\hline & Setembro/1996 & 28 & 22 & & 6,11 & 6,49 & \\
\hline & Outubro/1996 & 2 & 4 & & 0,89 & 1,22 & \\
\hline & Janeiro/1997 & 4 & 5 & & 1,94 & 3,08 & \\
\hline & Fevereiro/1997 & 2 & 1 & & 1,11 & 0,50 & \\
\hline & & & & 0,696 & & & 0.949 \\
\hline \multirow[t]{8}{*}{ Segundo } & Abril/1997 & 2 & 1 & & 1,70 & 0,55 & \\
\hline & Maio/1997 & 4 & 2 & & 2,71 & 1,29 & \\
\hline & Junho/1997 & 2 & 1 & & 2,03 & 1,09 & \\
\hline & Julho/1997 & 4 & 2 & & 3,86 & 2,16 & \\
\hline & Setembro/1997 & 6 & 2 & & 0,80 & 0,32 & \\
\hline & Outubro/1997 & 3 & 3 & & 0,45 & 0,50 & \\
\hline & Novembro/1997 & 5 & 1 & & 1,82 & 1,01 & \\
\hline & & & & 0,012 & & & 0,141 \\
\hline
\end{tabular}

Os valores da diversidade ( $\left.H^{\prime}\right)$ baseada no peso também foram reduzidos, em geral inferiores a 1,00 (Fig. 2A), com uma variação no RA de 0,25 (inverno/97) a 0,91 (verão/98), e na $\mathrm{AC}$ de 0 (verão/98) a 1,00 (primavera/97). Os valores inferiores de diversidade observados no RA durante grande parte do período de estudo, decorreram principalmente da predominância de peso de $R$. lalandii sobre as das demais espécies (Fig. 2B). A queda no verão/98 na AC, assim como observado para o número de indivíduos, ocorreu devido a presença de apenas uma espécie, $M$. higmani.

Quanto à similaridade baseada no número de indivíduos dos elasmobrânquios capturados no RA e na $\mathrm{AC}$, foram obtidos valores bastante elevados, variando de $50 \%$ (inverno/96) a $86,3 \%$ (outono/97). Considerando o peso como atributo numérico, tais valores também revelaram-se elevados, variando entre 12,9\% (inverno/96) e 93,5\% (verão/97). Observou-se uma tendência decrescente na similaridade entre as duas áreas (Fig. 3).

\section{DISCUSSÃO}

O tubarão $M$. higmani, pertencente à família Triakidae, apresentou um padrão semelhante de distribuição entre os dois sítios. Esta espécie tem sua alimentação baseada em crustáceos decápodas bentônicos e é comum em fundos de areia 

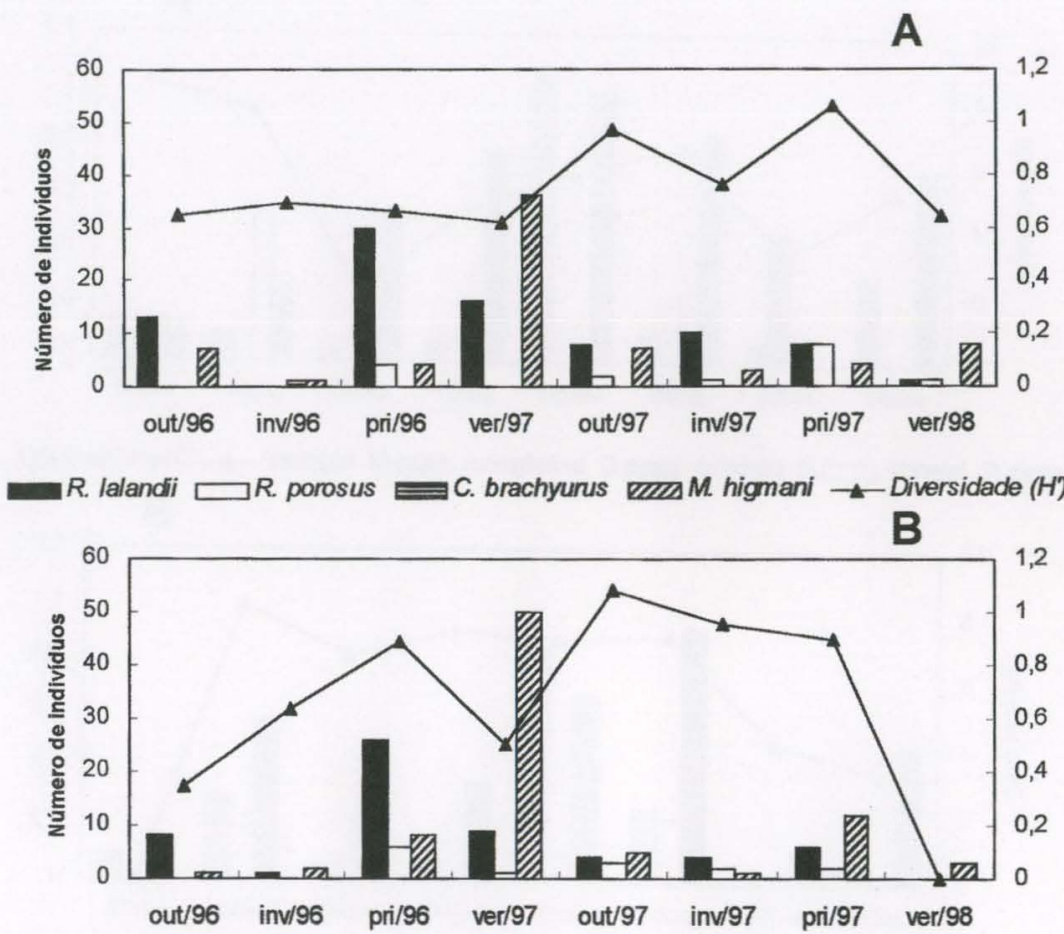

R. lalandi $\square R$. porosus wm M. higmani $₫$ Diversidade (H)

Fig. 1. Variação sazonal do número de indivíduos e da diversidade de espécies $\left(H^{\prime}\right)$ de tubarões costeiros capturados no recife artificial (A) e na área controle (B) (abr/96 a mar/98).

e lama, não associados à ambientes recifais (COMPAGNo 1984; HEEMSTRA 1997). Possivelmente estas características foram determinantes nos resultados observados, fazendo com que apesar de sua elevada captura no recife artificial, não seja considerada uma espécie atraída. Outros estudos que discutem a ocorrência de espécies deste gênero em recifes artificiais, tais como Mustelus antarticus (Günther, 1870) e Mustelus mustelus (Linnaeus, 1758), corroboram os resultados observados para M. higmani (BOMBACE et al. 1994; HAROUN et al. 1994; MCGLENNON \& BRANDEN 1994; SANTOS \& MONTEIRO 1998).

Dentre as quatro espécies de tubarões capturadas no presente estudo, três pertencem à família Carcharhinidae. Nela, estão as mais abundantes espécies de tubarões que ocorrem em águas tropicais e subtropicais. A maior parte das espécies desta família habita águas tropicais costeiras e de plataformas continentais, sendo que várias são comuns em recifes de corais e ilhas oceânicas, enquanto poucas são realmente oceânicas (COMPAGNO 1984).

Carcharhinus brachyurus atinge cerca de $2,9 \mathrm{~m}$ de comprimento total e possui hábito alimentar principalmente piscívoro, sendo encontrada próxima a recifes rochosos, em águas rasas de baías e em torno de ilhas oceânicas (MICHAEL 


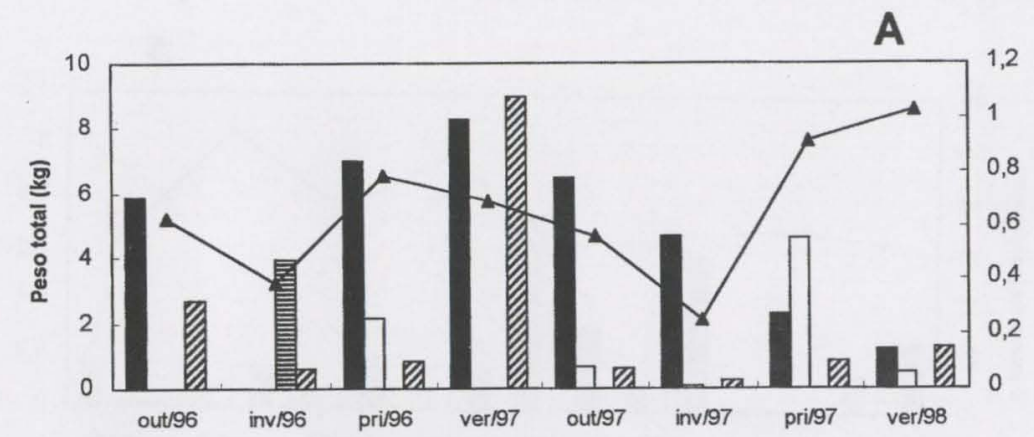

R. Ialandii $\square R$. porosus $\equiv$ C. brachyurus mm M. higmani $₫$ Diversidade $\left(H^{\prime}\right)$

B

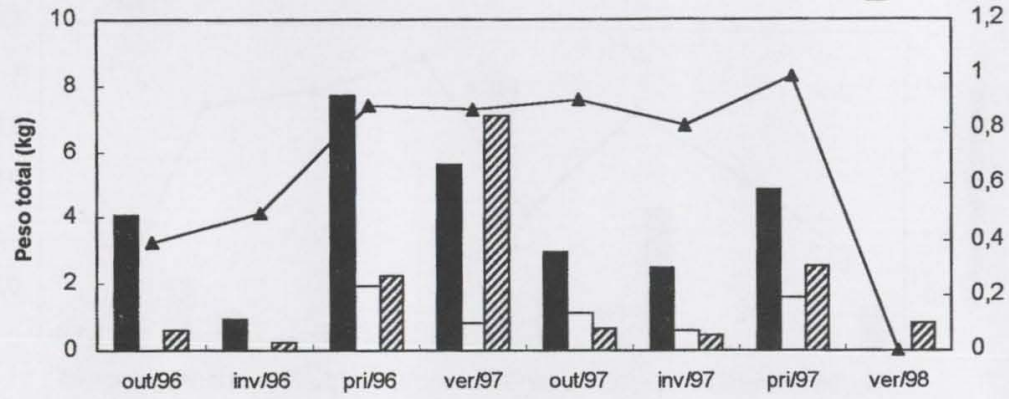

R. Ialandii $\square R$. porosus min M. higmani —-Diversidade $(\mathrm{H})$

Fig. 2. Variação sazonal do peso total $(\mathrm{kg})$ e da diversidade de espécies $\left(\mathrm{H}^{\prime}\right)$ de tubarões costeiros capturados no recife artificial (A) e na área controle (B) (abr/96 a mar/98).

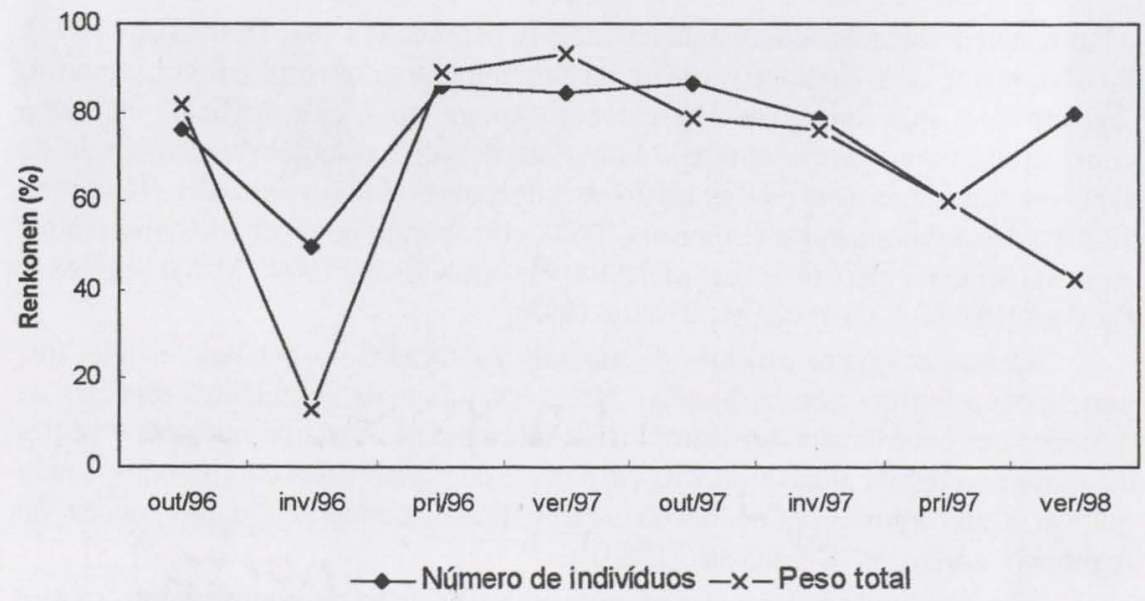

Fig. 3. Variação sazonal da similaridade (\%) baseada do número de individuos e no peso total $(\mathrm{kg})$ de tubarões costeiros capturados no recife artificial e na área controle (abr/96 a mar/98). 
1993). Apesar da associação desta espécie a ambientes recifais, a captura de um indivíduo juvenil deste tubarão na área de influência do recife artificial no presente estudo foi classificada como ocasional.

As outras duas espécies da família Carcharhinidae registradas no presente estudo, pertencem ao gênero Rhizoprionodon (Whitley, 1929), tipicamente cações de pequeno a médio porte, que habitam preferencialmente águas costeiras de regiões tropicais. São as únicas do gênero registradas para a costa brasileira, ambas ocorrendo em toda sua extensão (LESSA 1988).

A partir do segundo ano de estudo $R$. lalandii foi significativamente mais capturado na área de influência do recife artificial em termos de número de indivíduos. Isto foi refletido na tendência declinante na similaridade quantitativa entre os sítios. Apesar dos valores de peso de $R$. lalandii no recife e na área controle não diferirem significativamente, a evidência do aumento da captura desta espécie no recife artificial pode também ser considerada uma resposta ao incremento das estruturas em fevereiro/97.

Os valores inferiores de diversidade baseada no peso registrados no recife artificial durante praticamente todo período de estudo, evidenciam a maior abundância de $R$. lalandii em relação as outras na área de influência do recife artificial. HAROUN et al. (1994) e MCGLENNON \& BRANDEN (1994) também observaram uma maior diversidade em comunidades de peixes de áreas controle. Segundo estes autores, isto caracteriza uma tendência à dominância nos recifes artificiais por menor número de espécies.

A alimentação baseada em pequenos teleósteos pode ter sido o fator determinante da elevada captura de $R$. lalandii no recife artificial, visto que a predominância de teleósteos neste sítio, em relação a área controle, foi constatada por valores superiores de captura (ZALMON et al. 1999).

A amostragem em águas próximas à linha da costa pode ser responsável pela baixa captura de $R$. porosus, visto que esta espécie é pescada localmente em águas mais profundas (DI BENEDITTO et al. 1998).

\section{CONCLUSÕES}

As espécies de tubarões costeiros mais abundantes em capturas por rede de espera na região são Mustelus higmani (Triakidae) e Rhizoprionodon lalandii (Carcharhinidae).

O superior registro de Rhizoprionodon lalandii na área de influência do recife artificial, após o incremento de suas estruturas, sugere um potencial atrator desta espécie.

Os valores inferiores da diversidade (H') no recife artificial refletem a tendência à predominância de $R$. lalandii sobre as demais espécies neste sítio.

AGRADECIMENTOS. Os autores agradecem a Flavio A. Gomes, Eduardo G. Souza, Antônio

C. Pessanha, pescadores Eraldo e Jorge pelo apoio durante as amostragens. Agradecemos também as revisões críticas do manuscrito, realizadas por Carlos R. Ruiz-Miranda, Leandro R. Monteiro, Ana Paula Di Beneditto, Sérgio R. Floeter e dois revisores anônimos. Este estudo teve suporte financeiro do CNPq, FAPERJ e FENORTE. 


\section{REFERÊNCIAS BIBLIOGRÁFICAS}

AMBrose, R.F. \& S.L. SWARBRick. 1989. Comparison of fish assemblages on artificial and natural reefs off the coast of Southern California. Bull. Mar. Sci. 44: 718-733.

Baqueiro-Cardenas, E.; J. Hernández \& J. Aldana. 1999. Oil activity and artificial reefs programs in Campeche, Mexico. Proc. Seventh Internat. Conf. Artificial Reefs, Sanremo, p. 396-406.

Bombace, G.; G. Fabi; L. Fiorentin \& R. SPERAnZA. 1994. Analyses f the efficacy of artificial reefs located in five different areas of the Adriatic Sea. Bull. Mar. Sci. 55 (2-3): 559-580.

BonflL, R. 1994. Overview of world elasmobranch fisheries. FAO Fish. Techn. Paper (341): 1-119.

COMPAGNO, L.J.V. 1984. FAO species catalogue vol 4. sharks of the world. an annotated and illustrated catalogue of sharks species known to date. Part 2. FAO Fish. Synop. 4 (125): 251-655.

DI BENEDITTO, A.P.M.; R.M.A. RAmos \& N.R.W. LimA. 1998. Fishing ativity in Northern Rio de Janeiro State (Brazil) and its relation with small cetaceans. Brazil. Arch. Biol. Technol., Curitiba, 41 (3): 296-302.

D'ITRI, F.M. 1986. Artificial reefs - marine and freshwater applications. Chelsea, Lewis Publ. Inc., 589 p.

EKAU, W. \& B. KNOOPERS. 1999. An introduction to the pelagic system of the North-East and East Brazilian shelf. Arch. Fish. Mar. Res. 47 (2/3): 113-132.

FLOETER, S.R. \& A. SOARES-Gomes. 1999. Biogeographic and species richness patterns of Gastropoda on the Southwestern Atlantic. Rev. Brasil. Biol. 59 (4): 567-575.

haroun, R.J.; M. Gómez; J.J. Hernández; R. Herrera; D. Montero; T. Moreno; A. Portillo; M.E. TORRES \& E. SOLER. 1994. Environmental description of an artificial reef site in Gran Canaria (Canary Islands, Spain) prior to reef placement. Bull. Mar. Sci. 55 (2-3): 932-938.

HeEmStra, P.C. 1997. A review of the smooth-hound sharks (genus Mustelus, family Triakidae) of the Western Atlantic Ocean, with descriptions of two new species and a new subspecies. Bull. Mar. Sci. 60 (3): 894-928.

Holden, M.J. 1974. Problems in the rational exploitation of elasmobranch populations and some suggested solutions, p. 117-137. In: F.R. HARDEN-JONES (Ed.). Sea fisheries research. London, Elek Science, 510p.

LESSA, R.P. 1988. Premières observations sur la biologie reproductive de Rhizoprionodon lalandii (Valenciennes, 1839) (Pisces, Carcharhinidae) de la Côte Nord du Brésil - Maranhão. Rev. Brasil. Biol. 48 (4): 721-730.

McGlennon, D. \& K. L. Branden. 1994. Comparison of catch and recreational anglers fishing on artificial reefs and natural seabed in Gulf St. Vincent, South Australia. Bull. Mar. Sci. 55 (2-3): 510-523.

MiCHAEL, S.W. 1993. Reef sharks and rays of the world: a guide to their identification, ecology, and behaviour. Monterey, Sea Challengers, $\mathrm{VI}+107 \mathrm{p}$.

Morrisey, J.F. \& S.H. Gruber. 1993. Habitat selection by juvenile lemon sharks, Negaprion brevirostris. Environ. Biol. Fish. 38: 311-319.

PAL^C1O, F.J. 1982. Revisión zoogeografica marina del Sur del Brasil. Bol. Inst. Oceanogr. São Paulo 31 (1): 69-92.

Santos, M.N. \& C.C. Montelro. 1997. The Olhão artificial reef system (south Portugal): fish assemblages and fishing yield. Fish. Res. 30: 31-41.

- 1998. Comparison of the catch and fishing yield from an artificial reef system and neighbouring areas off Faro (Algarve, South Portugal): fish assemblages and fishing yield. Fish.

Res. 39: 55-65. 
Stevens, J.D.; R. BOnFlL; N.K. Dulvy \& P. Walker. 2000. The effects of fishing on sharks, rays and chimaeras (Chondrichthyans), and the implications for marine ecosystems. ICES Jour. Mar. Sci. 57: 476-494.

VALENTin, J.L. \& W.M. Monteiro-Ribas. 1993. Zooplankton community structure on the East-Southeast Brazilian continental shelf (18-23 S latitude). Continent. Shelf Res. 13 (4): 407-424.

ZALMON, I.R.; R. NOVELLI; M.P. GOMES \& V.V. FARIA. 1999. An artificial reef program on the Northern Coast of Rio de Janeiro, Brazil. Proc. Seventh Internat. Conf. Artificial Reefs, Sanremo, p. 105-112.

ZAR, J.H. 1999. Biostatistical analysis. New Jersey, Prentice-Hall, $4^{\text {th }}$ ed., 663p.

Recebido em 30.III.2001; aceito em 30.VII.2001. 